\title{
Deep Learning Based Predictive Compensation of Flicker, Voltage Dips, Harmonics and Interharmonics in Electric Arc Furnaces
}

This paper was downloaded from TechRxiv (https://www.techrxiv.org).

\section{LICENSE}

CC BY 4.0

SUBMISSION DATE / POSTED DATE

$30-12-2021 / 05-01-2022$

\section{CITATION}

Balouji, Ebrahim (2022): Deep Learning Based Predictive Compensation of Flicker, Voltage Dips, Harmonics and Interharmonics in Electric Arc Furnaces. TechRxiv. Preprint.

https://doi.org/10.36227/techrxiv.17707166.v1

$\mathrm{DOI}$

10.36227/techrxiv.17707166.v1 


\title{
Deep Learning Based Predictive Compensation of Flicker, Voltage Dips, Harmonics and Interharmonics in Electric Arc Furnaces
}

\author{
Ebrahim Balouji ${ }^{1}$, Özgül Salor ${ }^{2,3}$, Senior Member, IEEE, Tomas McKelvey ${ }^{1}$, Senior Member, IEEE \\ ${ }^{1}$ Chalmers University of Technology, Dept. of Electrical Engineering, Gothenburg, Sweden \\ ${ }^{2}$ Gazi University, Dept. of Electrical and Electronics Engineering, Ankara, Turkey \\ ${ }^{3}$ Kyrgyz-Turkish Manas University, Dept. of Electrical and Electronics Engineering, Bishkek, Kyrgyzstan \\ balouji@chalmers.se; salordurna@gazi.edu.tr; ozgul.salor@manas.edu.kg; tomas.mckelvey@chalmers.se
}

\begin{abstract}
In this research work, deep machine learning based methods together with a novel data augmentation are developed for predicting flicker, voltage dip, harmonics and interharmonics originating from highly time-varying electric arc furnace (EAF) currents and voltage. The aim with the prediction is to counteract both the response and reaction time delays of active power filters (APFs) specifically designed for electric arc furnaces (EAF). Multiple synchronous Reference frame (MSRF) analysis is used to decompose the frequency components of the EAF current and voltage waveforms into $d q o$ components. Then using lowpass filters and prediction of the future values of these $d q o$ components, reference signals for APFs are generated. Three different methods have been developed. In two of them, a lowpass Butterworth filter is used together with a linear FIR based prediction or long short-term memory network (LSTM) for prediction. In the third method, a deep convolutional neural network (CNN) combined with a LSTM network is used to filter and predict at the same time. For a $40 \mathrm{~ms}$ prediction horizon, the proposed methods provide $2.06 \%, 0.31 \%, 0.99 \%$ prediction errors of the $d q o$ components for the Butterworth and linear prediction, Butterworth and LSTM and CNN with LSTM, respectively. The error of the predicted reconstructed waveforms of flicker, harmonics, and interharmonics resulted in $8.5 \%$, $1.90 \%$, and $3.2 \%$ reconstruction errors for the above-mentioned methods. Finally, a Simulink and GPU based implementation of predictive APF using Butterworth filter + LSTM and a trivial APF resulted $96 \%$ and $60 \%$ efficiency on compensation of EAF current interharmonics.
\end{abstract}

Index Terms - active power filter, Butterworth filter, convolutional neural networks, deep learning, long short-term memory, linear prediction, multiple synchronous reference frame, electric arc furnace, flicker, harmonics, interharmonics, voltage dip.

\section{INTRODUCTION}

Electric arc furnaces (EAF) in an electricity network are often the largest consumers of electric power and hence the most power-quality (PQ)-threatening customers of the utility. For example, a single medium-sized EAF can induce a sudden demand for 100 MVA electric power. Such sudden peaks of energy demands repeated frequently at random intervals of a standard tap-to-tap time of an EAF, causes EAFs to be categorized as non-stationary loads, with undesired PQ events and variations such as flicker and voltage dips. Due to their operation principles, EAFs are also highly nonlinear loads, which draw currents from the power system rich in interharmonics and non-characteristic harmonics. These current harmonics often contribute significantly to the increase of voltage harmonics and interharmonics, in the transmission part of the power systems. Moreover, during resonance conditions of electric power systems, the amplitudes of these undesired frequency components can increase and damage or cause malfunction of some electronic devices. One of the most common solutions to such problematic issues is to employ active power filters (APF). These devices continuously make estimations of amplitudes and phases of the undesired frequency components and mitigated those components by supplying currents with the same amplitudes but half-cycleshifted phases. Hence, each regenerated current component should ideally be out-of-phase and exactly at the same amplitude so that perfect compensation is achieved at each harmonic or interharmonic frequency. Therefore, one of the most important key requirements in these types of methods is a fast and accurate estimation of the amplitudes and phases of the undesired frequency components, since inaccurate or delayed estimations could even cause amplification of the undesired current components. Having estimated the components to be mitigated, the second key requirement is a fast and accurate reaction of APFs hardware to supply the required mitigating currents to the system. Therefore improving the performances of APFs can be investigated in two main areas: algorithmic quality and hardware aspects. To improve the algorithmic quality, studies have been focused on reducing time delays of filters [1-3], reducing the computation times of the control algorithms [1], [4-7], and reducing the time spent on other algorithms $[8-11]$. The time delay due to the algorithms is called response time delay [12-17], whereas time delay caused by the hardware limitations is called reaction time delay [17-21]. For hardware improvement, researchers have tried to adapt faster data acquisition units [22, 24], more accurate and faster measurement sensors [25--27], to increase 
the switching frequency of power electronics switches [28-30] and to optimize LC filter parameters [31-35].

All these aforementioned studies provide valuable contributions; however, the contributions to algorithms to reduce response time delays remain limited. Moreover, solutions to the reaction time delays, such as generating the switches with the possibility of higher switching frequencies are quite expensive and limited. Using larger inductance and capacitance in LC filter design will help to mitigate the high-frequency harmonics and being less sensitive to impedance changes of the grid but this will in-turn add more delay to the overall reaction time of the APF. On the other hand, fast and more accurate data accusation units and sensors are also expensive and still, the most expensive ones have delays. Very limited studies had been proposed to tackle both algorithmic and hardware efficiency of APFs.

In this paper three different approaches have been employed to address the above-mentioned problems of EAF-current compensation by reducing the response time delay. A multiple synchronous Reference frame (MSRF) analysis is used to decompose the frequency components of the EAF current and voltage waveforms into $d q o$ components. Then using low-pass filters and prediction of the future values of these dqo components, reference signals for APFs are generated. Three different methods have been developed with the aim to reduce the response time delay. The first approach employ a low-pass Butterworth filter together with linear prediction. In the second approach, long short-term memory (LSTM) deep learning (DL) method together with a low-pass Butterworth filter is used With this method enough time can be gained to mitigate reaction and response time delays. In the final approach, a convolutional neural network (CNN) together with LSTM is proposed which combines the filter and prediction functions in one network. All of these approaches differ from the previous work of the authors in [36] such that the work in [36] mostly contributes on the mitigation of algorithmic delays only. The work presented in this paper proposes three different approaches to mitigate both algorithmic and hardware delays by increasing the prediction accuracy and horizon. Furthermore, besides predicting EAF current harmonics and interharmonics, solutions for efficient compensation of voltage fluctuations (flicker) and voltage dips originating from EAFs are also proposed. Finally, we extend the conference version of this paper [37] with implementation of the above mentioned methods on embedded computing system and an APF model in the Simulink environment. The results of employing the predictive methods in an APF are compared with a traditional non-predictive APF. The contributions and some important key-points of the three predictive methods presented in this research work can be listed as follows:

- With a novel proposed data augmentation method we can significantly increase the prediction accuracy

- Algorithmic and hardware delays can be mitigated thanks to the successful prediction of the future waveforms
- The methods are designed to compensate for several PQ problems at the same time, namely, harmonics, interharmonics, flicker, and voltage dips, based on the fact that they all appear to be interharmonics in the spectrum

- It is possible to do soft switching by predicting the dynamic voltage and current waveforms and this reduces the switching losses

- Since the filtering in the proposed method where CNN and LSTM are combined, there is no need to design an explicit low-pass filter

- Inductance and capacitance values of the LCL filter of the APF can be optimized and the problems coming from the grid side can be reduced. This can make it possible for APF/inverters to have less conduction losses and be less sensitive to network impedance changes and more important than that, to the frequency deviations of the electric power systems.

- A Virtual design of the ML based APF prototype is successfully implemented using the Simulink environment and embedded AI computing system Jetson TX2.

\section{EVERYTHING IS INTERHARMONICS}

\section{A. Flicker and voltage dip modeled as interharmonics}

The flicker phenomenon is an objectionable consequence of the random or periodic fluctuations on the voltage waveform envelope. These fluctuations have lower frequencies compared to the fundamental frequency and it can be shown that flicker appears as interharmonics around the fundamental and the harmonics in the frequency spectrum of the waveform [3841]. According to the standards IEEE 1159 and IEC 610004-15, flicker can be expressed as an amplitude modulation (AM) signal with a carrier frequency which corresponds to the fundamental frequency of the power system [42-44]

$$
\begin{aligned}
y(t) & =(A+m(t)) c(t) \\
& =\left(A+M \cos \left(w_{m} t+\phi\right)\right) \sin \left(w_{c} t\right)
\end{aligned}
$$

where $M$ and $w_{m}$ are the amplitude and frequency of the flicker fluctuation and $A$ and $w_{c}$ are the amplitude and frequency of the power system, respectively. Furthermore, $y(t)$ in (1) can be reformulated as,

$$
\begin{aligned}
y(t)=A \sin \left(w_{c} t\right)+ & \frac{M}{2}\left[\sin \left(\left(w_{c}+w_{m}\right) t+\phi\right)\right. \\
+ & \left.\sin \left(\left(w_{c}-w_{m}\right) t-\phi\right)\right] .
\end{aligned}
$$

As it can be seen in (2) the flickers which is the lower frequency fluctuation at $w_{m}$ of the fundamental amplitude, appears as interharmonics at frequencies $w_{c}-w_{m}$ and $w_{c}+w_{m}$, around the main component. Note that, flicker or any other fluctuation, does not need to be exactly sinusoidal shaped. However, it is also known that any envelope shape can also be modeled as the summation of various low frequency components. Then more number of frequency components will appear around the fundamental frequency, $w_{c}$. It can also be stated that the flicker frequency components will appear 
as interharmonics around the existing harmonics as well. For example in the case of a second harmonic in the system equation (2) can be formulated as,

$$
y(t)=\left(A+M \cos \left(w_{m} t+\phi\right)\right)\left[\sin \left(w_{c} t\right)+M_{2} \sin \left(2 w_{c} t\right)\right]
$$

and $y(t)$ can be expressed as

$$
\begin{aligned}
& y(t)=A \sin \left(w_{c} t\right)+A M_{2} \sin \left(2 w_{c} t\right) \\
& +\frac{M}{2}\left[\sin \left(\left(w_{c}+w_{m}\right) t+\phi\right)+\sin \left(\left(w_{c}-w_{m}\right) t+\phi\right)\right] \\
& +\frac{M M_{2}}{2}\left[\sin \left(\left(2 w_{c}+w_{m}\right) t+\phi\right)+\sin \left(\left(2 w_{c}-w_{m}\right) t+\phi\right)\right]
\end{aligned}
$$

where $A M_{2}$ corresponds to the amplitude of the second harmonic. Here, for the sake of simplicity it is assumed that the fundamental component and second harmonic have the same phase difference $\phi$ with respect to the flicker frequency component.

Furthermore, it can be stated that voltage dips can be modelled as changes of the waveform envelope in a random manner and that envelope can also be modelled as the modulation given in (1) with several modulating frequency components, whose summation forms the envelope. Therefore, it is possible to say that, a voltage dip will also appear as interharmonics around the fundamental and the existing harmonics. Hence, any method which quickly and accurately mitigates interharmonics around the fundamental frequency, will also compensate flicker and voltage dips. In order to be able compensate voltage dips, they should be predicted as soon as they start to appear in a few cycles of the fundamental and the APF should start compensating it by mitigating the related interharmonics immediately.

\section{B. A Solution and its Drawbacks}

In the past, many studies proposed solutions to estimate and compensate interharmonics [16], [45]. Only limited solutions have been presented for flicker compensation. To extract the interharmonics and harmonics components the proposed MSRF-based method in [11], [46] is used in this paper and is illustrated in Fig 1 For example, to extract the 45 $\mathrm{Hz}$ frequency components, $w$ in $\mathrm{Fig} 1$ needs to be defined as $w=2 \pi 45$. In the extraction process of the phase and amplitude of the $45 \mathrm{~Hz}$ frequency component in the dqo frame, the low-pass filter block is a key component in the analysis chain and is needed due to time variation together with the effect of other frequency components in the signal, e.g. the blue signal shown in Fig. 2. After low-pass filtering of the $d q o$ frame component, the reverse operation $d q o^{-1}$ can be used to obtain the pure $45 \mathrm{~Hz}$ component.

The choice of LP-filter in the MSRF analysis chain is important to obtain high performance. One of the most wellknown choices in the industry is the Butterworth filter for lowpass filtering of $d q o$ components. In this research work, a 4th order Butterworth filter with $1.5 \mathrm{~Hz}$ cutoff frequency is used for low-pass filtering and and an example of using this filter on filtering of $d^{+}$component is shown in Fig. 2. The drawback

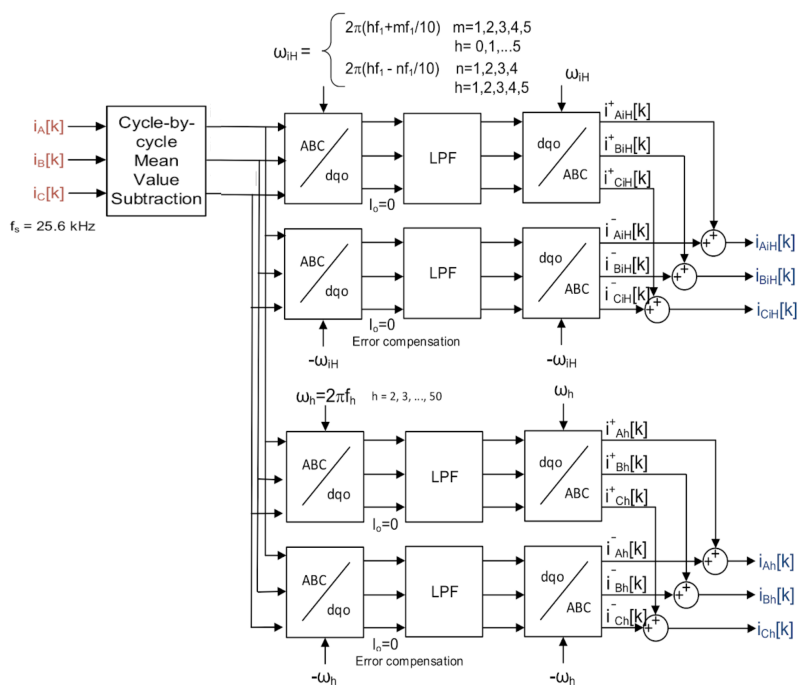

Fig. 1: Structure of the MSRF method used to decompose three-phase waveforms into its frequency components for both positive and negative $d q o$ frames.

of a this causal Butterworth filter is that it has a settling time delay of almost 0.8 cycle of the fundamental frequency $(16 \mathrm{~ms}$ for $50.0 \mathrm{~Hz}$ ) in following the trend of the $d^{+}$components. This delay is measured by comparing the results obtained using a zero-phase digital filter (in MATLAB called filtfilt) which implements the non-causal filter and is only applicable for off-line analysis [47]. Later these values will be used as the desired true values for training ML methods to predict the future values of $d q o$ components. The settling time delay of the Butterworth filter is one part of the total algorithmic delay. Since hardware delays also exist it is desirable to be able to provide prediction which are even further ahead than the delays incurred by the casual Butterworth filter. In the next section, three possible solutions for mitigating these delays are addressed.

\section{Deep Machine Learning for Predictive FILTERING}

In this section, the three proposed approaches are explained for predictive low-pass filtering of $d q o$ components illustrated in Fig. 11 The overall schemata of the proposed solutions are illustrated in Fig. 3 In $P S_{1}$, we use low-pass Butterworth filter to filter out the unwanted frequency components. Following this step, linear prediction is used to estimate the future samples of the zero-phase filtered $d q o$. Similarly, in $P S_{2}$ we use LSTM to predict the future values of the zerophase filtered $d q o$ values. The aim of using linear prediction and LSTM is to predict far ahead to both mitigate the lag of the Butterworth filter and contributes to cancelling the rest of algorithmic delays and reaction time delay of APFs. Note that the same Butterworth filter defined in III-B is used as low-pass filter in $P S_{1}$ and $P S_{2}$. In $P S_{3}$, a combination of 

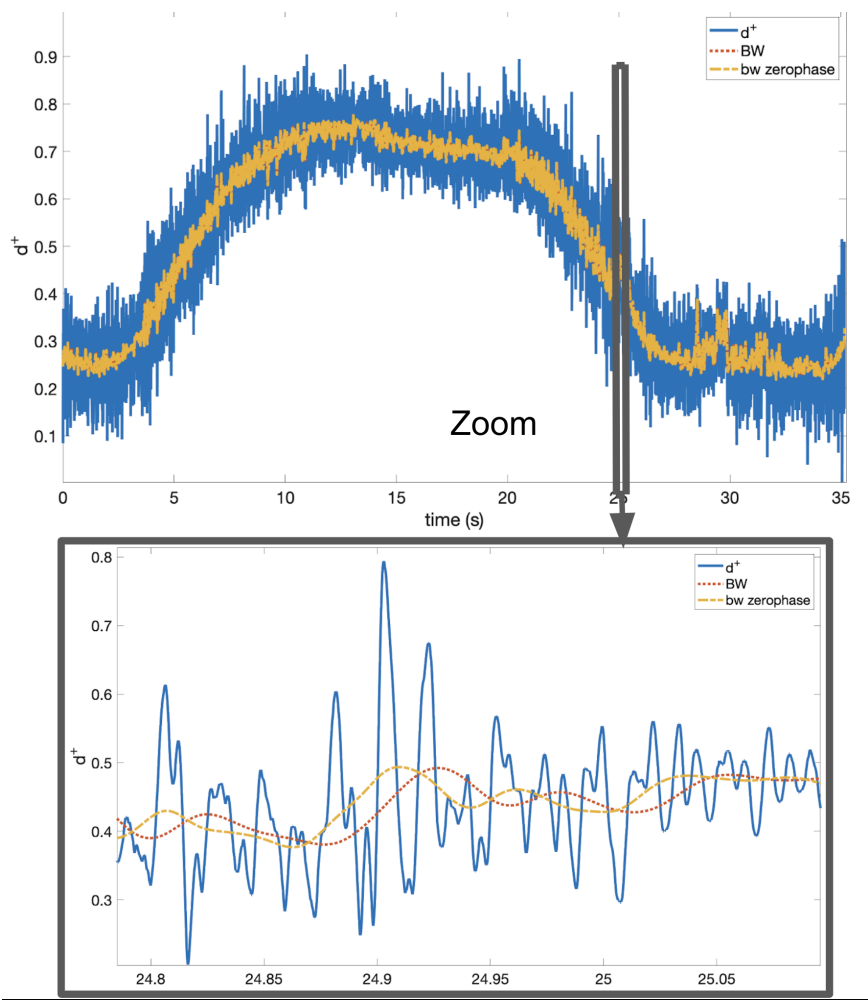

Fig. 2: The example of $d^{+}$component for $f_{i H}=45 \mathrm{~Hz}$ component with its low-pass filtered version using Butterworth filtering and zero-phase Butterworth filter (using filtfilt of Matlab).

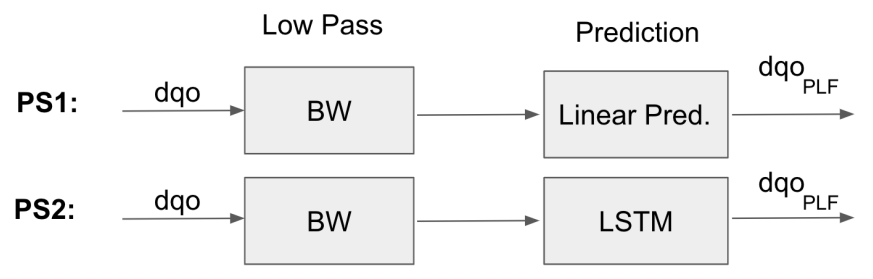

Low pass + prediction

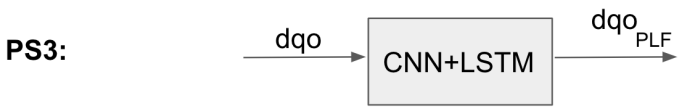

Fig. 3: Proposed solutions developed in this research work. PS and PLF stand for Proposed Solution and Predictive Low-pass Filter respectively.

CNN and LSTM networks are employed to do the filtering and prediction task at the same time. The theory of these methods will be explained in detail in the next subsections. Please note that all notations for proposed method carried out on current waveform of three-phase $\operatorname{EAF}\left(i_{a}, i_{b}, i_{c}\right)$ and it will be the same for voltage waveform if $\left(v_{a}, v_{b}, v_{c}\right)$ are considered instead.

\section{A. Low-Pass Predictor for Butterworth Linear Prediction}

The linear prediction method aims to forecast the amplitude of the zero-phase low-pass filtered $d q o$ components at time $t$ by a FIR filter if length $M$ which provide a weighted sum of the $M$ past samples from the output of the casual Butterworth filter [48]. For example, predicting the $N^{\text {th }}$ future sample of the Butterworth low-pass filtered $d^{+B W}$ component can be defined as:

$$
d^{+\hat{B} W}(t+N)=\sum_{k=1}^{M} h_{k} d^{+B W}(t-k)
$$

where $h_{k}$ are the coefficients of the linear predictor and $B W$ is notation of Butterworth filter. These coefficients are obtained from training data by solving the least-squares problem:

$$
\min _{\left\{h_{k}\right\}_{k=1}^{M}} \sum_{t}\left[\left(d_{z p}^{+B W}(t+N)-\sum_{k=1}^{M} h_{k} d^{+B W}(t-k)\right)^{2}\right]
$$

where $d_{z p}^{+B W}(t)$ is the signal obtained from the anti-causal zero-phase filtering using filtfilt. Note that the linear prediction coefficients are obtained by training on the output of the Butterworth filter shown in Fig. 3 and the desired output are future samples of the zero-phase filter output. Later these coefficients will be used in low-pass filtering and prediction in the MSRF method, which can be seen in pseudo-code in Algorithm 1 .

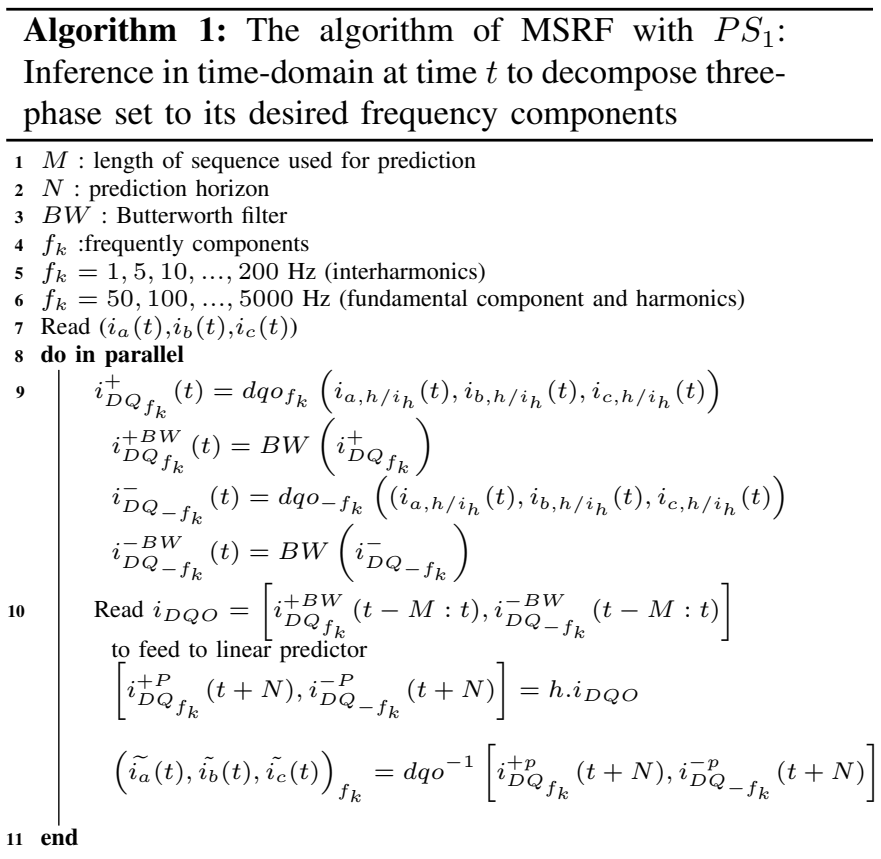




\section{B. Butterworth LSTM based low-pass predictor}

In this approach, different from the previous solution, structure of LSTM allows to use a novel data augmentation step. Simply, the selected window of data is flipped from buffer and concatenated with itself:

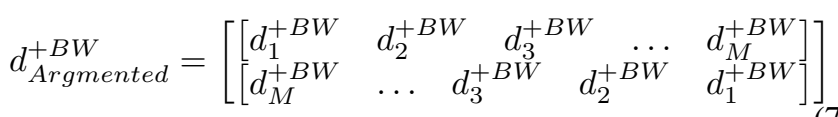

This augmentation will act as regularizer and can be helpful to increase the prediction robustness and accuracy. Another difference from linear prediction is that LSTM will provide a nonlinear prediction function [49], [50]. In the training phase, at each time step, we select a window of data with the length of $M$ together with the future value of $d q o$ from the zerophase digital filter, i.e. $d_{z p}^{+B W}(t+N)$, and fed it to the LSTM networks by augmentation. By using chain derivative and Adam optimizer [51] for gradient upgrade the weights on each layer of LSTM cells will be trained. With this style, it can create a model of patterns by trained weights and can predict the future values of $d q o$ component. Fig. 4 illustrates the basic schema of LSTM Cells. The stateful operator of each cell is shown by $h$ that is obtained using some operations on $d^{+B W}$ at index $n$, and $h_{n-1}$ and information of previous cells $C_{n}$. The cell has three gates to control the information flow. The forget gate $f_{n}$ controls if the previous state of the cell should be erased or kept. The input gate $i_{n}$ controls how much information from other cells should be added to the current cell. The output gate $o_{n}$ controls the information that goes on to the next cells. Note that the computed states $h$ are fed to the LSTM cells in the next LSTM layer. The obtained states $h_{1, \ldots, n}$ from the while loop of Algorithm 2 in the last layer of the LSTM are fed to fully connected layers (FC). In the fully connected layers these extracted states $\left(h_{1}, h_{2}, \ldots, h_{n}\right)$ ) are used to obtain the future value of $d q o$ components. The process of using the $P S_{2}$ in the MSRF method is addressed in Algorithm 2

\section{CNNLSTM based low-pass predictor}

In this case of study, we use the same data augmentation suggested in the previous subsection as a regularizer to increase the prediction accuracy and result robustness. Also, different from $P S_{1}$ and $P S_{2}$ in $P S_{3}$ we use a combination of CNN and LSTM to not only low-pass filter the $d q o$ components but also predict the future value of them. The idea is to use the CNN to filter selected window of the $d q o$ components data with zero delays and represent them with a smaller number of features [52], [53]. This will help the LSTM and deep neural network in FC layers to see the highlighted features of the selected sequence. The detail of this solution together with MSRF is given in Algorithm 3 . We select several filters and a window of data from $d q o$ components after MSRF inversion (see Fig. 1) and convolve

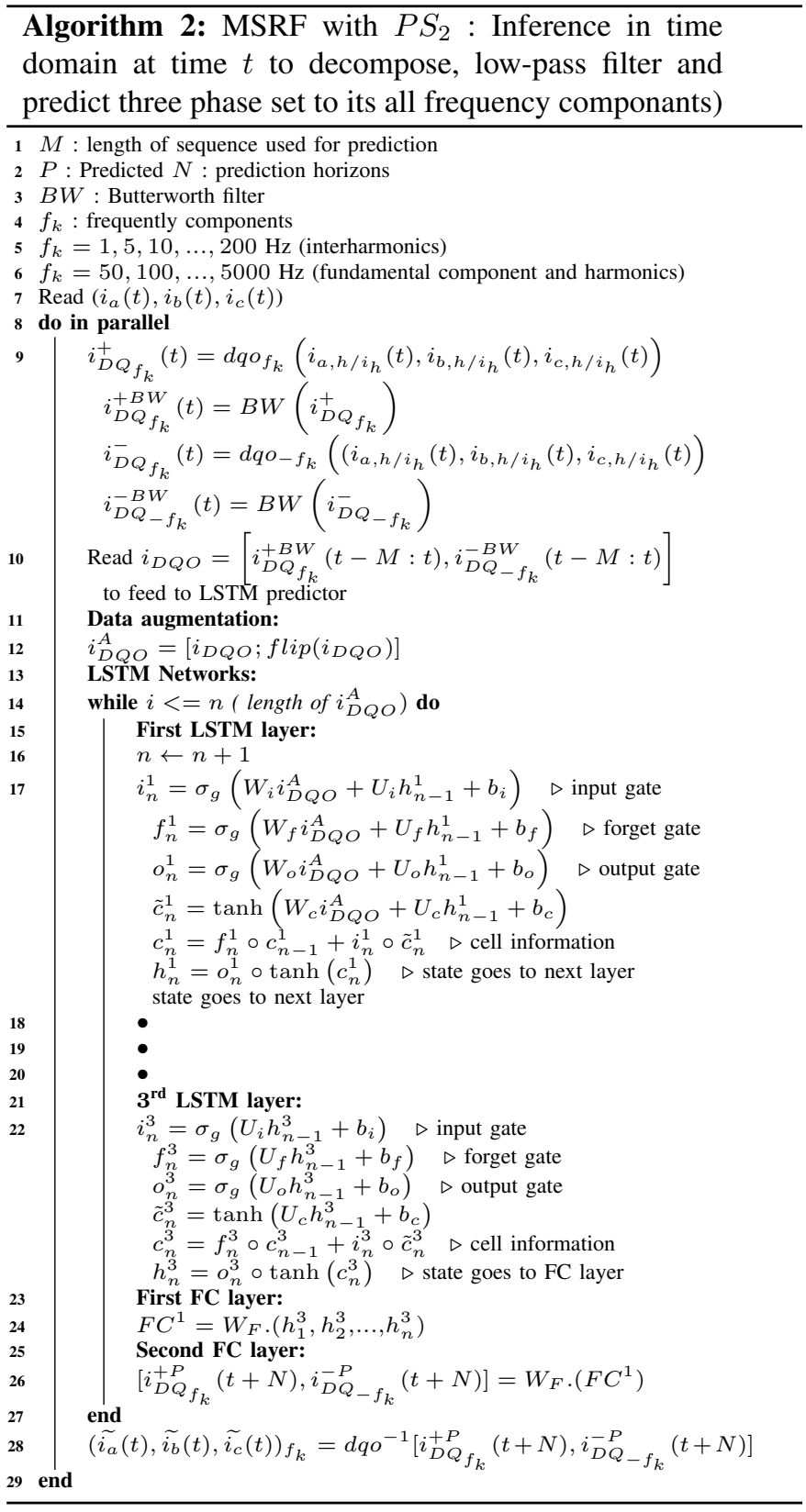

the initialized weights of the filters through the selected sequence. Then the averagePool will obtain the average of multiplication of wights to data on each filter and pass them to the next layer. Note that since we are striding the wights in the convolution we simply lowering the dimension of the input sequence at the same time. In the second step, the extracted features by $\mathrm{CNN}$ are fed to the deep neural network to create a new sequence with the desired length (preferably considerably smaller than the selected window of $d q o$ component). The extracted features which are smoother version of $d q o$ components and matched to downsampled and smoother version of the timeline in the raw $d q o$ component. 


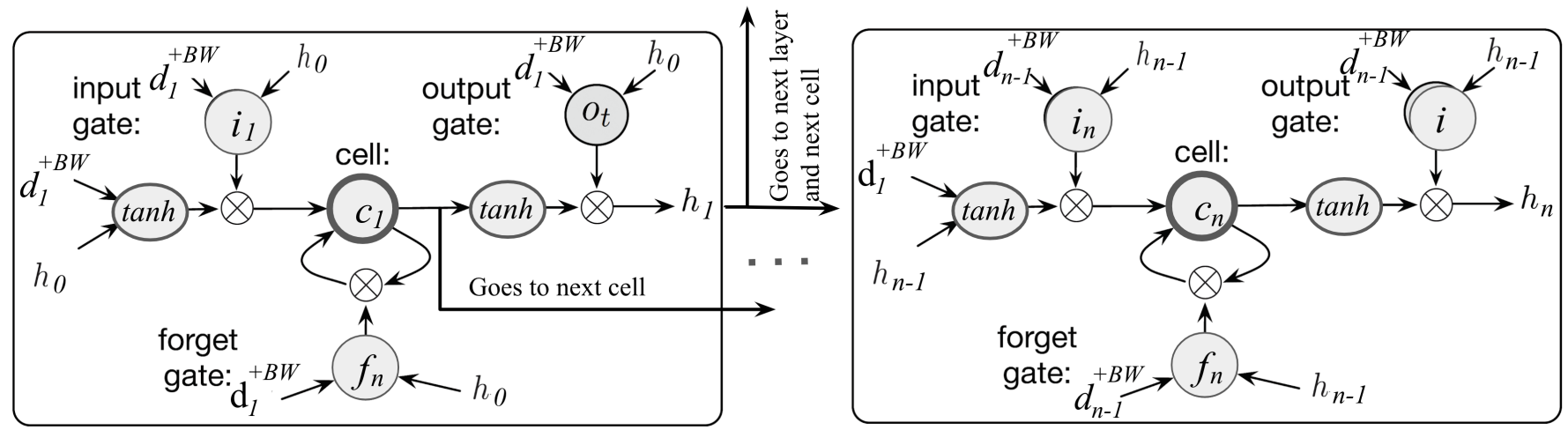

Fig. 4: The unrolled diagram of LSTM cell representing the gates defined in the text. $d^{B W+}, h$ and $c$ stands for input data, state and cell of LSTM respectively.

Finally, LSTM sequentially analyzes the extracted features to do the prediction task.

\section{Application of Proposed Solutions}

The proposed methods are implemented in a Python software environment using a workstation with an Intel i7 3.40 GHz CPU, 48GB RAM, and an NVIDIA Titan XP 12GB GPU. The proposed methods are trained and tested on 35 GB of data collected from four transformer substations feeding EAFs. The recordings were carried out at a $25.6 \mathrm{kHz}$ sampling rate from $154 \mathrm{kV}$ transmission lines between two to three hours duration from each substation. We have done two different kinds of training and testing of the proposed algorithms. In the first scenario, $80 \%$ of the data is used for training and $20 \%$ for testing. To test the robustness of the proposed algorithms we also the train the networks on data from three separate EAFs and test on the data set from the last EAF in a cross-validation format. The three methods are evaluated by averaging the prediction error results over the resulting four sets of models. The overall results of the proposed solutions in the previous section are presented in Table I. The results are based on the $d^{+}$component for the $45 \mathrm{~Hz}$ interharmonic extracted for both voltage and current waveforms of the EAF and the prediction horizon is set to $40 \mathrm{~ms}$. It can be seen that in training with cross-validation we have a slight increase in prediction error rate. It can also be seen that $P S_{2}$ perform best of the three approaches. Compared with $P S_{2}$, the prediction error increases slightly for the $P S_{3}$ approach where CNN and LSTM are integrated. In $P S_{1}$ Linear prediction of low-pass filtered $d^{+}$component combined with low-pass filtering has the highest prediction error. An investigation of the relation between the prediction horizon with respect to the prediction error is shown in Fig 5 . The prediction error increases approximately exponentially for all three methods. In Table I the decrease in percentage points of the prediction error for the proposed data augmentation methods is shown. The augmentation reduces the prediction error significantly for $P S_{2}$ and $P S_{3}$. This can be because of the way the weights are trained towards modeling the data to give the lowest prediction error. In another word, in the prediction tasks, when data is nonlinear and complex, the weights cannot model the trend of the desired signal and can end up be trained to just pass along the last value of the selected data point. But flipping data and feeding with the original window of selected data will act as a regularizer which makes the weights to train in a way that they are focused on learning the pattern of the window of data and predict future value. Finally, to see the prediction accuracy and delays mentioned earlier, for different proposed solutions we show results from the prediction of $d^{+}$component for the second harmonic in Fig. 6. The prediction is carried out on one second of data (25600 samples) recorded from the boring phase of EAF. Note that the amplitude of the $d^{+}$component is normalized with the max-min method to keep the values between zero and one. The sequence is the same zoomed sequence illustrated in Fig. 2. To avoid clutter in the figures we refrained from plotting the non-filtered $d^{+}$components. From Fig. 6 it is clear that the $P S_{1}$ method has a higher prediction error compared to $P S_{2}$ and $P S_{3}$. The method $P S_{1}$ can follow the trend of the desired signal but with a slight delay. As it can be seen $P S_{2}$ follow the pattern of the desired signal of the zero-phase filtered $d^{+}$component very well. Recall from subsection III-B, the desired signal are the future values of zero-phase Butterworth filter obtained by filtfilt command of Matlab. For the case of $P S_{3}$ the prediction error and delay is lower than the $P S_{1}$ but higher than $P S_{2}$.

\section{DISCUSSION ON COMPENSATION OF FLICKER, INTERHARMONICS, HARMONICS AND VOLTAGE DIPS}

\section{A. Flicker and Voltage Dip}

As it is addressed in Section $[1$ it is observed that a voltage fluctuation with $5-\mathrm{Hz}$ frequency can generate flicker and it can be expressed as interharmonics with 45 and $55 \mathrm{~Hz}$ frequency. Therefore for flicker compensation, it is needed to compensate 


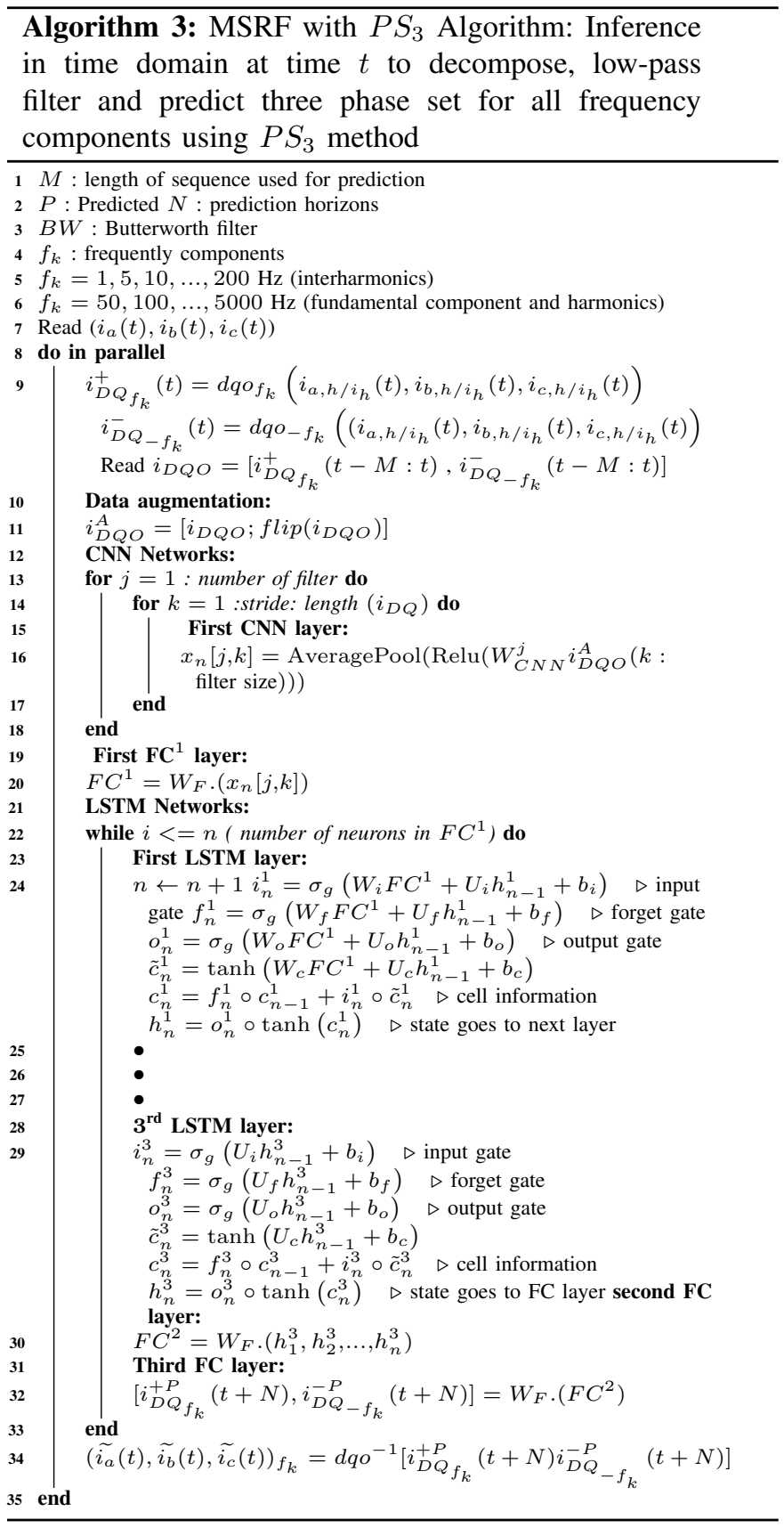

the interharmonics around the fundamental frequency component and those around harmonics up to the forth. Thus, it is aimed to estimate or predict future values of interharmonics for mitigation of the delays in flicker compensation. Similarly, one can also do predictions on fundamental frequency components and predict the voltage dip and compensate it in real-time. A waveform with considerable flicker followed by a momentary voltage dip originating from EAF from field measurements is shown in Fig. 7] with the blue curve together with the reconstructed waveform and using the $P S_{2}$ prediction method. Since reconstruction of future voltage waveform can
TABLE I: Investigation of proposed solutions and their results for $40 \mathrm{~ms}$ prediction horizon of $d^{+}$component of $45 \mathrm{~Hz}$ interharmonics of EAF current and voltage. Abbreviations used on this table: prediction (Pred.), error (Err.), validation (Val.)

\begin{tabular}{lllll}
\hline \hline Scenario $\backslash$ Methods & $d^{+}$ & $P S_{1}$ & $P S_{2}$ & $P S_{3}$ \\
\hline $\mathbf{4 0}$ ms Pred. Err. (\%) & $d_{i}^{+}$ & 2.06 & 0.32 & 0.99 \\
$\mathbf{8 0}$ (\%) Training 20 (\%) Val. & $d_{v}^{+}$ & 1.91 & 0.30 & 0.82 \\
\hline Data augmentation & $d_{i}^{+}$ & - & 0.15 & 0.69 \\
effect on Pred. Err. (\% points) & $d_{v}^{+}$ & - & 0.41 & 0.83 \\
\hline $\mathbf{4 0}$ ms Pred. Err. (\%) & $d_{i}^{+}$ & 2.32 & 0.42 & 1.22 \\
Cross Val. & $d_{v}^{+}$ & 2.01 & 0.37 & 0.89 \\
\hline Waveform Pred. Err. (\%) & $d_{i}^{+}$ & 7.59 & 1.92 & 3.5 \\
with 40 ms prediction & $d_{v}^{+}$ & 8.59 & 1.5 & 3.01 \\
\hline \hline
\end{tabular}

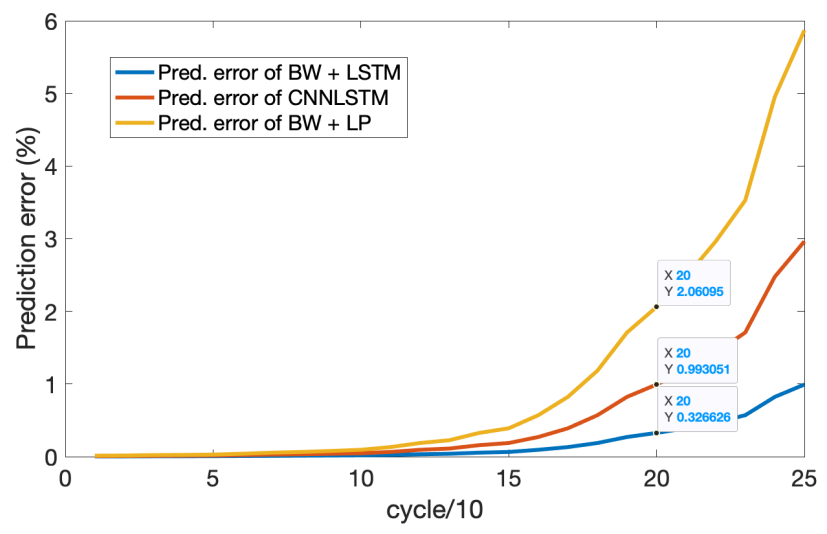

Fig. 5: Investigation of prediction error for $d_{i}^{+}$as a function of the prediction horizon for proposed solutions. Prediction horizon increased every cycle/10

be accomplished it shows that it is possible to filter out the fluctuations by injecting the reference signal with out of phase for flicker compensation. For the case of a voltage dip, the injection will be the same phase as the fundamental component. To illustrate the prediction accuracy, the envelope of both $V_{a}$ and the predicted version of it are also shown in the figure. It can be seen that the predicted signal can follow the trend of the original $V_{a}$ two cycles ahead and the reconstruction error is $1.35 \%$ for the selected segment of data in the figure.

\section{B. Harmonics and Interharmonics in current}

Similar to the case of voltage flicker, in this subsection a reconstruction of the current waveform of an EAF using MSRF based on $P S_{2}$ is presented. The reconstruction of current waveform based on predicted frequency components resulted with $1.79 \%$ error rate on selected window of data shown in Fig. 8. Since the current waveform of the EAF has more stochastic behavior compared to the voltage, all of the proposed solutions resulted slightly less accurate pre- 


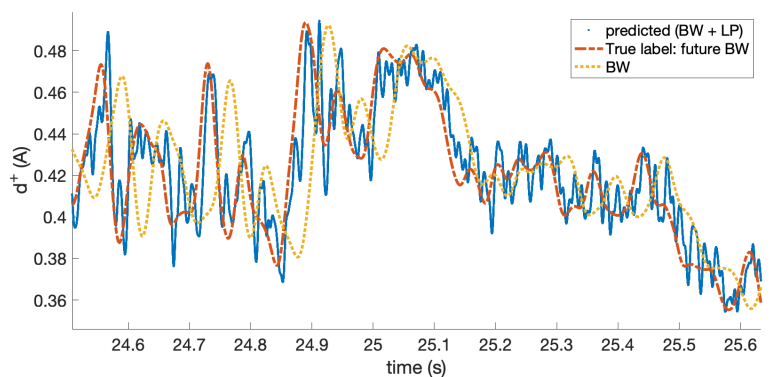

(a)

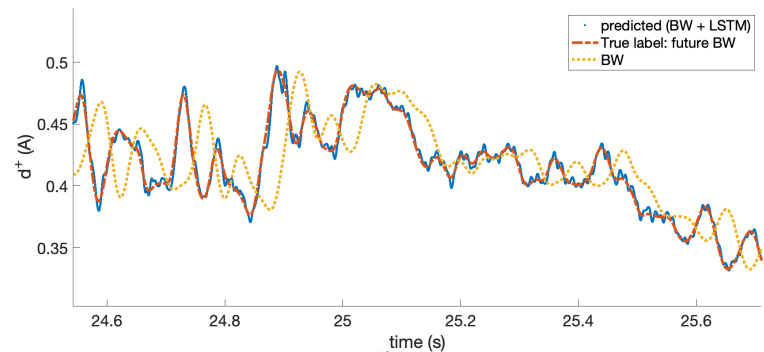

(b)

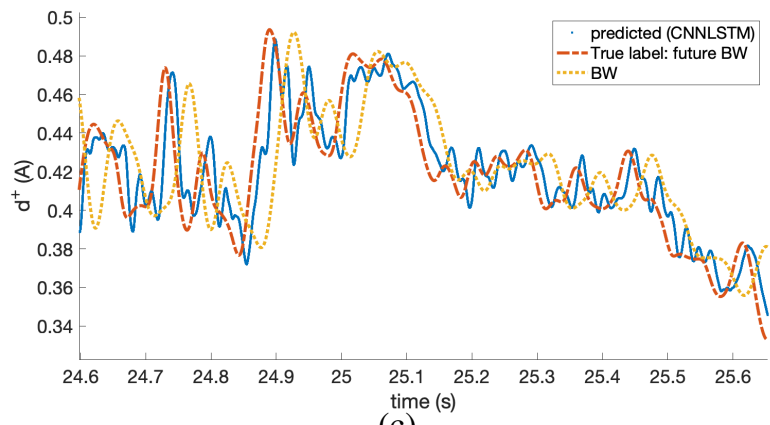

(c)

Fig. 6: Example of prediction of $d^{+}$component of second harmonic using the three proposed solutions. (a) $P S_{1}$ (b) $P S_{2}$ (c) $P S_{3}$.

dictability. The reconstruction error rates of current waveform using $P S_{1}$ and $P S_{3}$ are $7.22 \%$ and $2.99 \%$ respectively. The accuracy of reconstruction using $P S_{2}$ still has highest accuracy for a prediction horizon of $40 \mathrm{~ms}$. The error on the reconstructed current waveform is slightly higher than the average prediction error of the $45 \mathrm{~Hz}$ frequency component only. This can be reasoned around possible overlap in estimation of different frequency components and slight increase in prediction error of high frequency components such as the $9^{t h}$ harmonic. To avoid to keep the presentation clear, only the reconstruction results of $i_{a}$ using $P S_{2}$ is shown in Fig. 8 where the reconstructed waveform is generated by summing up the fundamental component and the components from the $2^{\text {nd }}$ harmonic, the odd harmonics up to the $10^{\text {th }}$, and interharmonics with a $5 \mathrm{~Hz}$ resolution up to $200 \mathrm{~Hz}$. It can be seen that the predicted and reconstructed $i_{a}$ follow the pattern of the original $i_{a}$ with a good accuracy before (40 $\mathrm{ms}$ ) it appears and the delay effect of the Butterworth filter

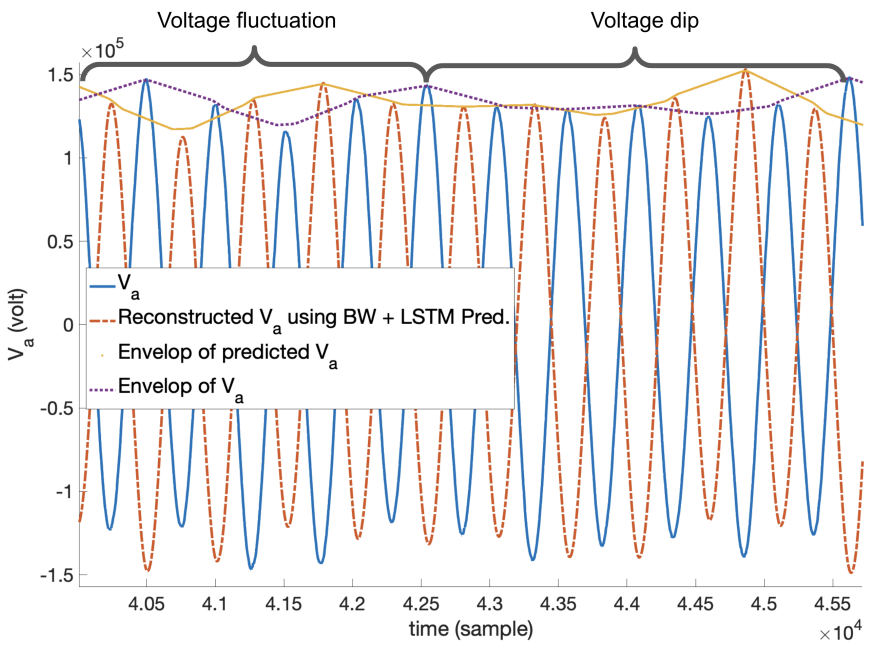

Fig. 7: Comparison of the original voltage waveform with predicted reconstructed voltage waveform by summing up the estimated frequency components using time domain $P S_{2}$ (the fundamental, the $2^{\text {nd }}$ harmonic, the odd harmonics up to $10^{t h}$, and interharmonics with $5-\mathrm{Hz}$ resolution up to $200 \mathrm{~Hz}$ )

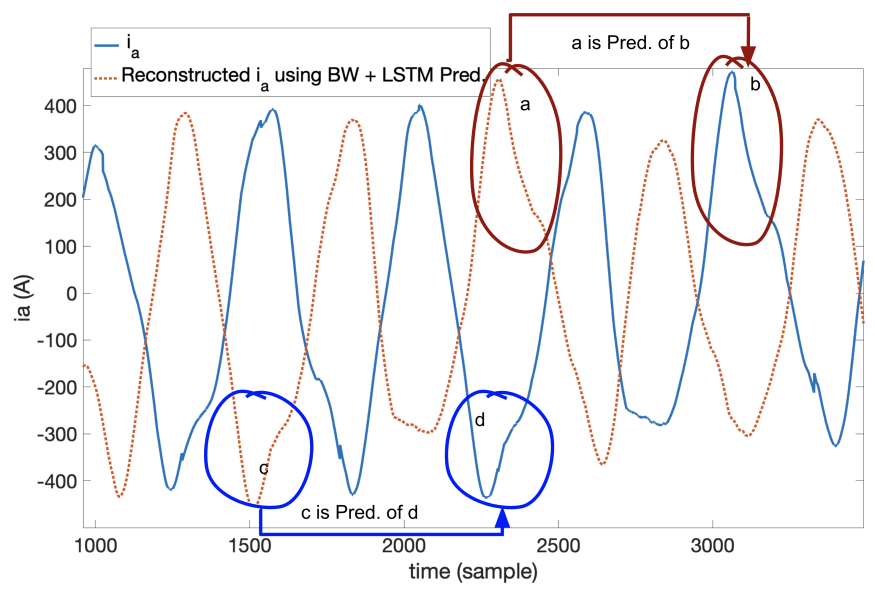

Fig. 8: Comparison of the original current waveform with predicted reconstructed current waveform by summing up the estimated frequency components using $P S_{2}$ (the fundamental, the $2^{\text {nd }}$ harmonic, the odd harmonics up to the $10^{t h}$, and interharmonics with a $5 \mathrm{~Hz}$ resolution up to $200 \mathrm{~Hz}$ )

is mitigated. Thus this extra available time contributes to the mitigation of the rest of reaction time delay and the response time delay of the APF.

\section{Implementation of APF in Simulink and methods embed- ded system}

In this subsection, a virtual prototype of ML based and a trivial APF is implemented. The aim is to evaluate the performance and effectiveness of proposed predictive algorithms mentioned in previous sections. The $P S_{2}$ based approach 
is used for the prediction and low-pass filtering task in Simulink since, as shown in the previous sections, it gave the best performance. The block schema and specification of the developed shunt APF in Simulink environment is illustrated in Fig. 9.

The Simulink model consists of three main power electronics-based blocks including $A C$ grid, EAF, and Shunt Active filter. To make sure the model is close to the real environment we used data that is recorded from transformers feeding an EAF and give it as the reference signal to a six gate IGBT bridge with a $P I$ controller. This block will act as a current source that regenerates the EAF current. We also replicated the AC grid block diagram using a similar six gates $I G B T$ bridge. The voltage in the AC grid block has to have a constant level $(400 \mathrm{KV})$ and be in phase with EAF to avoid the effect phase overlap resulting in the extra frequency deviation problems. Hence, the reference current waveform is obtained based the difference of the reference voltage $(400 \mathrm{KV})$ and measured voltage from EAF. Note that this is an example model of compensating interharmonics and harmonics including variation in the fundamental component and models specifications for the purpose of flicker and voltage dip compensation. With a successful compensation, $R_{L}$ in Fig. 9 will have a pure sinusoidal current flow. Two scenarios are defined to show the effect of the predictive based approaches versus a trivial APF solution. The first scenario is an implementation of a trivial APF where the reference signal is estimated with only MSRF analysis + Butterworth filter. In the second scenario the reference signal is predicted using MSRF $+P S_{2}$. In both scenarios, at first, the voltages and currents of EAF together with voltage and current of AC grid are measured and sent to GPU accelerated processor (Jetson TX2) via TCP link using Ethernet cable. In the second step, we estimate the $d q$ components using the MSRF box. Then in predictive APF, $P S_{2}$ is used for low-pass filtering and $40 \mathrm{~ms}$ prediction of the future value of the $d q$ components. Note that this $40 \mathrm{~ms}$ delay originates from the lag of the Butterworth filter mentioned in II-B (16 ms), PI controller response time $(21 \mathrm{~ms})$, the data communication delay between Simulink and Jetson TX2 (2.5 ms), actuation of APF (0.8 ms) and processing time of data on MSRF blocks and $P S_{2}(0.2$ $\mathrm{ms})$. Furthermore, the reference signal is generated using by $M S R F^{-1}$ on predicted and low-pass filtered values of the $d q$ components. Similar approach is carried out for the trivial APF scenario but the only difference is that the there is no LSTM for prediction of the filtered $d q$ components. As an example, one minute of EAF data is generated by the EAF block with the APF generated signal for the two scenarios. The MSRF decomposition includes frequency components from $45 \mathrm{~Hz}$ to $55 \mathrm{~Hz}$ with $1 \mathrm{~Hz}$ resolution. The EAF current and the compensation results on $0.9 \mathrm{~s}$ of data between $1.1 \mathrm{~s}$ to $1.8 \mathrm{~s}$ using the trivial APF and predictive APF with $P S_{2}$ are illustrated in Fig. 10 (a) and Fig. 10 (b) respectively.

The results shown in Figure 10 (a) and (b) clearly illustrate the importance and value of a predictive-based approach. The performance evaluation on the selected window of data where we measure the remaining undesired frequency components after compensation resulted in $60 \%$ and $96 \%$ efficiency for the trivial and $P S_{2}$ based APF respectively. The trivial APF reduce the undesired frequency components at a lower compensation efficiency. However, during transients it can cause an increase in the interharmonics which is evident from Figure 10 (a) at time $>1.7 \mathrm{~s}$. The reason is that in the case of highly time-varying EAF current and voltage, the amplitude and the phase of frequency components are varying almost every cycle. Thus, having a $40 \mathrm{~ms}$ delay means that the APF compensates the interharmonics based on measurements two cycle ago which are no longer valid at present time.

\section{CONCLUSIONS}

In this research work, it is shown that it is possible to predict highly time-varying voltage and current waveforms of EAFs up to two cycles $(40 \mathrm{~ms}$ ) of the fundamental frequency using a deep-learning based method. This is to provide reference signals to active power filters (APFs) so that harmonics, interharmonics, flicker and voltage dips can be mitigated. The key point is choosing the right prediction method and pre-processing steps which is decomposing the waveform into enough number sinusoidal components. These sinusoidal signals are represented with $d q o$ frames using the MSRF method. It has been shown that $d q o$ frame analysis after lowpass filtering of $d q o$ components became smoother and less time-varying over time. Hence, it is thus easier to predict the $d q o$ components rather then the current or voltage waveforms directly. Three prediction methods are investigated. $P S_{1}$ is a combination of low-pass filtering and linear prediction. $P S_{2}$ is a combination of low-pass filtering and non-linear prediction using an LSTM model. $P S_{3}$ is a combination of CNN and LSTM architectures which together perform the prediction without an explicit low-pass filter. For the $P S_{2}$ and $P S_{3}$ solutions a particular data augmentation is employed, which is flipping the selected window of data and feed it together with the original window, can act as regularization and increase the prediction robustness and decrease the prediction error. It has been shown that the flicker and voltage dip phenomenons can be represented as interharmonic problems and they can be compensated and predicted along with compensating harmonics and interharmonics of both voltage and current waveform of EAFs. Finally, it can be concluded that each of the proposed methods have their own pros and cons. Implementation of $P S_{1}$ is easy and less complicated but its average prediction error is almost seven times and two times larger compared to $P S_{2}$ and $P S_{3}$, respectively. This can be an essential factor when it comes to predicting highly time-varying signals such as EAF current and voltage waveform. The $P S_{3}$ made it possible to develop a DL-based low-pass filtering which mitigates the need for tuning of Butterworth filter and automatically extract the future values of low-pass filtered $d q o$ components. 


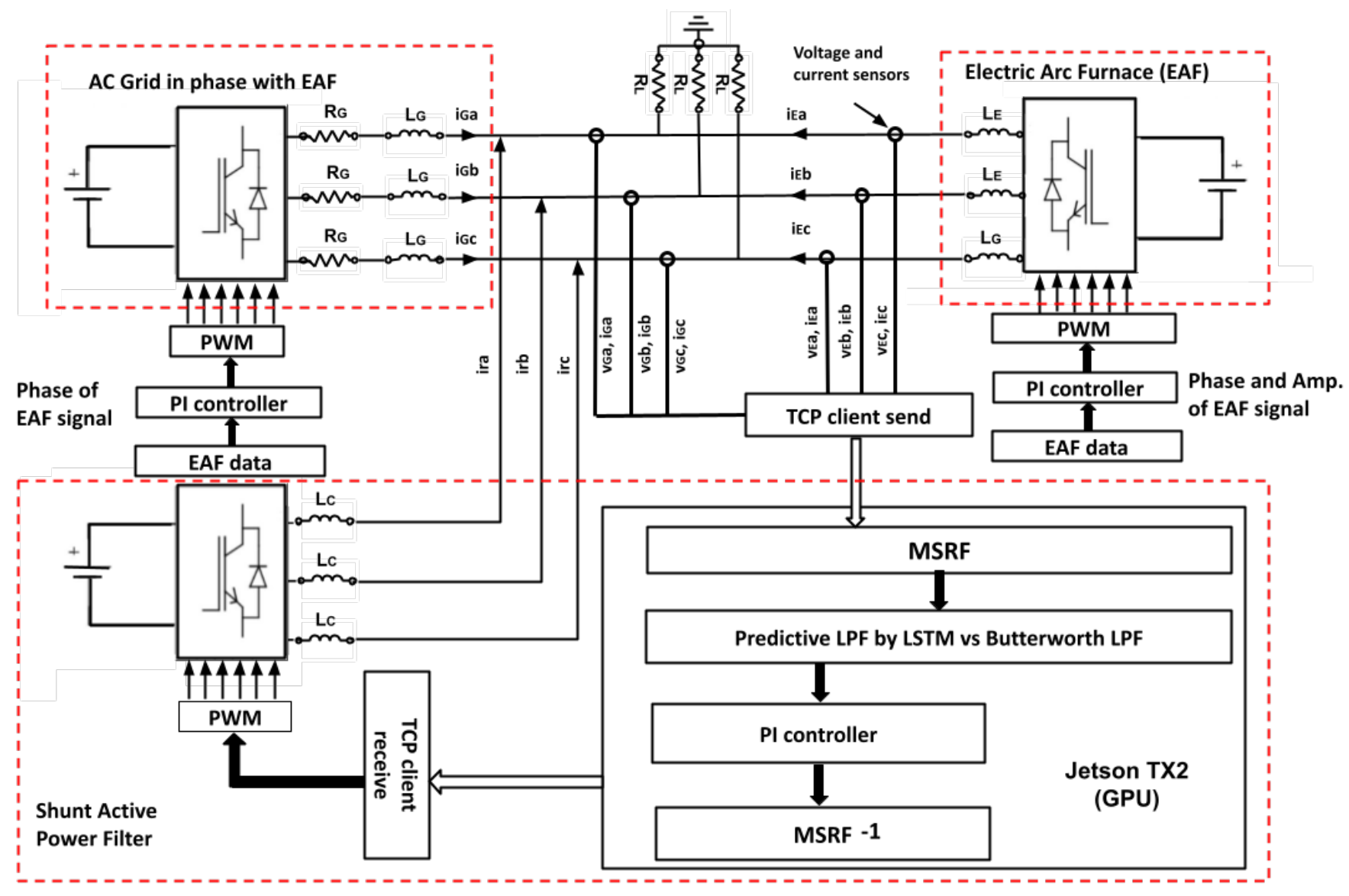

Fig. 9: GPU accelerated Simulink based implementation of a trivial and ML based shunt APF. The elements used in Simulink can be listed as: $R_{G}=1 \Omega, R_{L}=10 \mathrm{k} \Omega$ and $L_{E}=L_{G}=L_{C}=3 \mathrm{mH}$.

Finally, we observed that the implementation of predictive based methods on a replicated electric power system using Simulink improve the performance compared to a a trivial APFs by more than $36 \%$.

\section{ACKNOWLEDGEMENTS}

The authors would like to thank the Chalmers University of Technology for financially supporting this research work. The authors would also like to thank the Public Research Institutions and Development Projects Support Group (KAMAG) of the Scientific and Technological Research Council of Turkey (TUBITAK) for providing EAF field data.

\section{REFERENCES}

[1] L. Asiminoael, F. Blaabjerg, and S. Hansen, "Detection is key-harmonic detection methods for active power filter applications," IEEE Industry Applications Magazine, vol. 13, no. 4, pp. 22-33, 2007.

[2] P. S. B. Nascimento, H. E. P. de Souza, F. A. S. Neves, and L. R. Limongi, "FPGA implementation of the generalized delayed signal cancelation-phase locked loop method for detecting harmonic sequence components in three-phase signals," IEEE Transactions on Industrial Electronics, vol. 60, no. 2, pp. 645-658, 2013.
[3] E. U. Logoglu, O. Salor, and M. Ermis, "Real time detection of interharmonics and harmonics of AC electric arc furnances on GPU framework," IEEE Transactions on Industry Applications, 2019.

[4] H. F. Blanchette, T. Ould-Bachir, and J. P. David, "A state-space modeling approach for the FPGA-based real-time simulation of high switching frequency power converters," IEEE Transactions on Industrial Electronics, vol. 59, no. 12, pp. 4555-4567, 2012.

[5] Z. Shu, S. Xie, and Q. Li, "Single-phase back-to-back converter for active power balancing, reactive power compensation, and harmonic filtering in traction power system," IEEE Transactions on Power Electronics, vol. 26, no. 2, pp. 334-343, 2011.

[6] Z. Shu, M. Liu, L. Zhao, S. Song, Q. Zhou, and X. He, "Predictive harmonic control and its optimal digital implementation for mmcbased active power filter," IEEE Transactions on Industrial Electronics, vol. 63, no. 8, pp. 5244-5254, 2016.

[7] G. Becerra-Nuñez, A. Castillo-Atoche, J. Vazquez-Castillo, A. Datta, R. G. Quijano-Cetina, R. Peña-Alzola, R. Carrasco-Alvarez, and E. Osorio-De-La-Rosa, "An FPGA Kalman-MPPT implementation adapted in sst-based dual active bridge converters for DC microgrids systems," IEEE Access, vol. 8, pp. 202 946-202 957, 2020.

[8] Z. Shu, Y. Guo, and J. Lian, "Steady-state and dynamic study of active power filter with efficient FPGA-based control algorithm," IEEE Transactions on Industrial Electronics, vol. 55, no. 4, pp. 1527-1536, 2008.

[9] N. Dai, M. Wong, F. Ng, and Y. Han, "A FPGA-based generalized pulse width modulator for three-leg center-split and four-leg voltage source inverters," IEEE Transactions on Power Electronics, vol. 23, no. 3, pp. 1472-1484, 2008. 


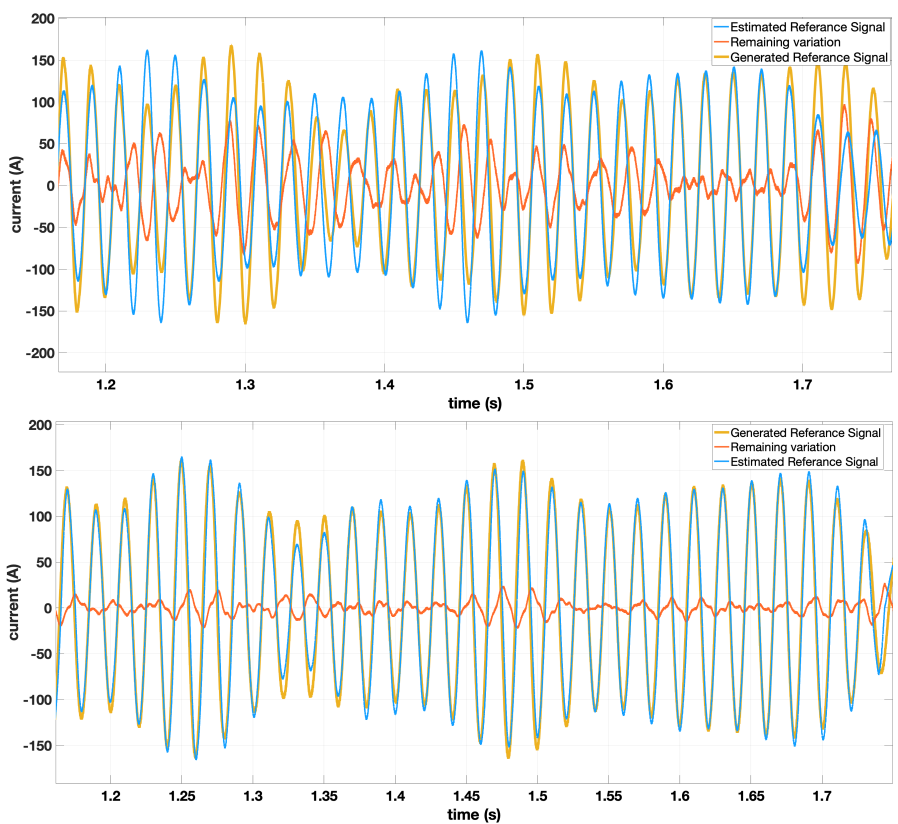

(b)

Fig. 10: Comparison of the result of the Simulink based implementation of a trivial APF versus ML based APF. (a) Compensation of the frequency components between 45:1:55 $\mathrm{Hz}$ without prediction. (b) Compensation of the frequency components between $45: 1: 55 \mathrm{~Hz}$ with $P S_{2}$ of $d q$ components.

[10] E. Uz-Logoglu, O. Salor, and M. Ermis, "Real-time detection of interharmonics and harmonics of AC electric arc furnaces on GPU framework," in 2017 IEEE Industry Applications Society Annual Meeting. IEEE, 2017, pp. 1-8.

[11] E. Balouji, Ö. Salor, and M. Ermis, "Exponential smoothing of multiple reference frame components with GPUs for real-time detection of time-varying harmonics and interharmonics of EAF currents," IEEE Transactions on Industry Applications, vol. 54, no. 6, pp. 6566-6575, 2018.

[12] L. Asiminoaei, F. Blaabjerg, and S. Hansen, "Evaluation of harmonic detection methods for active power filter applications," in Twentieth Annual IEEE Applied Power Electronics Conference and Exposition, 2005. APEC 2005., vol. 1, 2005, pp. 635-641 Vol. 1.

[13] T. Fang, Q. Zhou, F. Ding, X. Wu, Z. Li, and H. Tang, "Response time of reactive power based on different definitions and algorithms," Journal of Modern Power Systems and Clean Energy, vol. 9, no. 2, pp. 440-449, 2021.

[14] M. A. A. Mohd Zainuri, M. A. Mohd Radzi, A. Che Soh, N. Mariun, N. Abd Rahim, J. Teh, and C.-M. Lai, "Photovoltaic integrated shunt active power filter with simpler adaline algorithm for current harmonic extraction," Energies, vol. 11, no. 5, p. 1152, 2018.

[15] M. A. M. Radzi and N. A. Rahim, "Neural network and bandless hysteresis approach to control switched capacitor active power filter for reduction of harmonics," IEEE Transactions on Industrial Electronics, vol. 56, no. 5, pp. 1477-1484, 2009.

[16] M. El-Habrouk, M. Darwish, and P. Mehta, "Active power filters: A review," in IEEE Electric Power Applications, vol. 147, no. 5. IEEE, 2000, pp. 403-413.

[17] s.a. ABB n.v. Power Quality Products, "Active filters - PQFI-PQFMPQFS improving power quality for efficiency and reliability," 2018.

[18] R. Martinek, J. Rzidky, R. Jaros, P. Bilik, and M. Ladrova, "Least mean squares and recursive least squares algorithms for total harmonic distortion reduction using shunt active power filter control," Energies, vol. 12, no. 8, p. 1545, 2019.
[19] J. Tyster et al., "Power semiconductor nonlinearities in active du/dt output filtering," 2014.

[20] E. Balouji, K. BäckstrÖm, T. McKelvey, and O. Salor, "Deep-learningbased harmonics and interharmonics predetection designed for compensating significantly time-varying EAF currents," IEEE Transactions on Industry Applications, vol. 56, no. 3, pp. 3250-3260, 2020.

[21] E. Balouji, "On machine learning based signal processing for power system applications," Ph.D. dissertation, Department of Electrical Engineering, Chalmers University of Technology, 2020.

[22] S. A. O. da Silva, P. F. Donoso-Garcia, P. C. Cortizo, and P. F. Seixas, "A three-phase line-interactive ups system implementation with seriesparallel active power-line conditioning capabilities," IEEE Transactions on Industry Applications, vol. 38, no. 6, pp. 1581-1590, 2002.

[23] M. Cirrincione, M. Pucci, and G. Vitale, "A single-phase DG generation unit with shunt active power filter capability by adaptive neural filtering," IEEE Transactions on Industrial Electronics, vol. 55, no. 5, pp. 2093-2110, 2008

[24] M. Odavic, M. Sumner, and P. Zanchetta, "Control of a multi-level active shunt power filter for more electric aircraft," in 2009 13th European Conference on Power Electronics and Applications, 2009, pp. $1-10$.

[25] S. Ziegler, R. C. Woodward, H. H. Iu, and L. J. Borle, "Current sensing techniques: A review," IEEE Sensors Journal, vol. 9, no. 4, pp. 354-376, 2009.

[26] J. D. Bull, N. A. F. Jaeger, and F. Rahmatian, "A new hybrid current sensor for high-voltage applications," IEEE Transactions on Power Delivery, vol. 20, no. 1, pp. 32-38, 2005.

[27] F. Rahmatian and A. Ortega, "Applications of optical current and voltage sensors in high-voltage systems," in 2006 IEEE/PES Transmission Distribution Conference and Exposition: Latin America, 2006, pp. 1-4.

[28] M. J. Rogers, E. R. Motto, and M. Steiner, "Performance comparison of state-of-the-art $300 \mathrm{a} / 1700 \mathrm{v}$ si igbt and $\mathrm{SiC}$ mosfet power modules," IEEE Power Electronics Magazine, vol. 7, no. 3, pp. 44-51, 2020.

[29] A. Pal and K. Basu, "A novel modulation strategy for active rectification of a snubber less soft-switched single stage 30 high frequency link DC-AC converter," in 2017 IEEE PES Asia-Pacific Power and Energy Engineering Conference (APPEEC), 2017, pp. 1-10.

[30] M. Rodríguez, A. Rodríguez, P. F. Miaja, D. G. Lamar, and J. S. Zúniga, "An insight into the switching process of power mosfets: An improved analytical losses model," IEEE Transactions on Power Electronics, vol. 25, no. 6, pp. 1626-1640, 2010.

[31] V. Blasko and V. Kaura, "A novel control to actively damp resonance in input LC filter of a three-phase voltage source converter," IEEE Transactions on Industry Applications, vol. 33, no. 2, pp. 542-550, 1997.

[32] H. Park and J. Jung, "Power stage and feedback loop design for LLC resonant converter in high-switching-frequency operation," IEEE Transactions on Power Electronics, vol. 32, no. 10, pp. 7770-7782, 2017.

[33] Y. W. Li, "Control and resonance damping of voltage-source and current-source converters with LC filters," IEEE Transactions on Industrial Electronics, vol. 56, no. 5, pp. 1511-1521, 2009.

[34] J. He and Y. W. Li, "Generalized closed-loop control schemes with embedded virtual impedances for voltage source converters with LC or LCL filters," IEEE Transactions on Power Electronics, vol. 27, no. 4, pp. 1850-1861, 2012.

[35] C. Gong, W. K. Sou, and C. S. Lam, "Design and analysis of vector proportional-integral current controller for LC-coupling hybrid active power filter with minimum DC-link voltage," IEEE Transactions on Power Electronics, pp. 1-1, 2021.

[36] E. Balouji, K. Bäckström, T. McKelvey, and Ö. Salor, "Deep-learningbased harmonics and interharmonics predetection designed for compensating significantly time-varying EAF currents," IEEE Transactions on Industry Applications, vol. 56, no. 3, pp. 3250-3260, 2020.

[37] E. Balouji, O. Salor, and T. Mckelvey, "Predictive compensation of EAF flicker, voltage dips harmonics and interharmonics using deep learning," in 2021 IEEE Industry Applications Society Annual Meeting. IEEE, 2021.

[38] O. Salor, B. Gultekin, S. Buhan, B. Boyrazoglu, T. Inan, T. Atalik, A. Acik, A. Terciyanli, O. Unsar, E. Altintas, Y. Akkaya, E. Ozdemirci, 
I. Cadirci, and M. Ermis, "Electrical power quality of iron and steel industry in Turkey," in 2007 IEEE Industry Applications Annual Meeting, 2007, pp. 404-423.

[39] J. A. Pomilio and S. M. Deckmann, "Flicker produced by harmonics modulation," IEEE Power Engineering Review, vol. 22, no. 8, pp. 6767, 2002.

[40] T. Tayjasanant, Wencong Wang, Chun $\mathrm{Li}$, and Wilsun $\mathrm{Xu}$, "Interharmonic-flicker curves," IEEE Transactions on Power Delivery, vol. 20, no. 2, pp. 1017-1024, 2005.

[41] T. Keppler, N. R. Watson, J. Arrillaga, and Shiun Chen, "Theoretical assessment of light flicker caused by sub- and interharmonic frequencies," IEEE Transactions on Power Delivery, vol. 18, no. 1, pp. 329-333, 2003.

[42] "IEEE recommended practice for monitoring electric power quality," IEEE Std 1159-2019 (Revision of IEEE Std 1159-2009), pp. 1-98, 2019.

[43] "IEEE recommended practice-adoption of IEC 61000-4-15:2010, electromagnetic compatibility (emc)-testing and measurement techniquesflickermeter-functional and design specifications," IEEE Std 1453-2011, pp. $1-58,2011$.

[44] E. Balouji and O. Salor, "Digital realisation of the IEC flickermeter using root mean square of the voltage waveform," IET Generation, Transmission \& Distribution, vol. 10, no. 7, pp. 1663-1670, 2016.

[45] B. Singh, K. Al-Haddad, and A. Chandra, "A review of active filters for power quality improvement," IEEE Transactions on Industrial Electronics, vol. 46, no. 5, pp. 960-971, 1999.

[46] E. Uz-Logoglu, O. Salor, and M. Ermis, "Online characterization of interharmonics and harmonics of AC electric arc furnaces by multiple synchronous reference frame analysis," in 2015 IEEE Industry Applications Society Annual Meeting. IEEE, 2015, pp. 1-11.

[47] L. B. Jackson, Digital Filters and Signal Processing: With MATLAB® Exercises. Springer Science \& Business Media, 2013.

[48] S. V. Vaseghi, Advanced digital signal processing and noise reduction. John Wiley \& Sons, 2008.

[49] S. Hochreiter and J. Schmidhuber, "Long short-term memory," Neural computation, vol. 9, no. 8, pp. 1735-1780, 1997.

[50] C. Olah, "Understanding lstm networks," 2015.

[51] D. P. Kingma and J. Ba, "Adam: A method for stochastic optimization," arXiv preprint arXiv:1412.6980, 2014.

[52] A. Krizhevsky, I. Sutskever, and G. E. Hinton, "Imagenet classification with deep convolutional neural networks," Advances in neural information processing systems, vol. 25, pp. 1097-1105, 2012.

[53] J. Donahue, L. A. Hendricks, M. Rohrbach, S. Venugopalan, S. Guadarrama, K. Saenko, and T. Darrell, "Long-term recurrent convolutional networks for visual recognition and description," IEEE Transactions on Pattern Analysis and Machine Intelligence, vol. 39, no. 4, pp. 677-691, 2017.

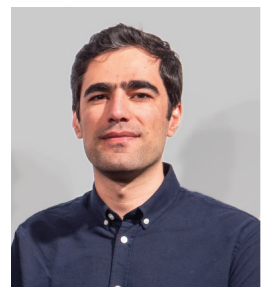

Ebrahim Balouji (S'13) received M.Sc degree in electrical and electronics engineering from Gazi University, 2015. From 2015-2017 he worked as a researcher of Turkey's National Power Quality Project. Since 2017, he has researched analyzing power system data using machine learning and signal processing tools towards his Ph.D. studies in the electrical engineering department of Chalmers University of Technology, Gothenburg, Sweden. He received Gunnar Engström honer reward from ABB foundation for his research in 2021

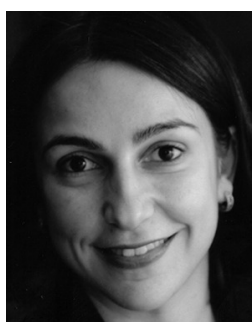

Özgül Salor (S'98-M'05-SM'18) received the B.Sc., M.Sc., and Ph.D. degrees from Middle East Technical University, Ankara, Turkey, in 1997, 1999, and 2005, respectively, all in electrical and electronics engineering. From 2001 to 2003, she was a Professional Researcher with the Center for Spoken Language Research, University of Colorado, Boulder, CO, USA. From 2006 to 2012, she was with the Power Electronics Department, Space Technologies Research Institute, Scientific and Technological Research Council of Turkey. Since 2012, she has been a Professor with the Department of Electrical and Electronics Engineering, Gazi University, Ankara, Turkey. Her current research interests include machine learning techniques and signal processing for power quality analysis. Currently, she is with the Kyrgyz-Turkish Manas University in Kyrgyzstan to start the new department of Electrical and Electronics Engineering since August 2019. Dr. Salor was the recipient of the Outstanding Paper Award and Certificate of Recognition Award from the Metal Industry Committee of the IEEE Industry Applications Society in 2009 and 2015 and the Meritorious Paper Award of MIC in 2013 and 2019.

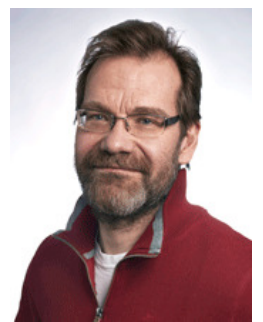

Tomas McKelvey received the M.Sc. degree in Electrical Engineering from Lund University, Lund, Sweden, in 1991, and the Ph.D. degree in Automatic Control from Linköping University, Linköping, Sweden, in 1995. He held research and teaching positions with Linköping University from 1995 to 1999, where he became a Docent in 1999. From 1999 and 2000, he was a Visiting Researcher with the University of Newcastle, Newcastle, NSW, Australia. Since 2000, he has been with the Chalmers University of Technology, Gothenburg, where he has held a Full Professor position and has been the head of the Signal Processing Group since 2006 and 2011, respectively. His current research interests include model-based and statistical signal processing, system identification, and optimal control with applications to radar systems, electrical power systems, and vehicle propulsion systems. systems. 